\title{
Análise da aplicabilidade da Portaria 557 do Ministério das Cidades em estudos de viabilidade técnico-econômica para incineração com recuperação energética de resíduo sólido urbano no Brasil
}

\author{
Analysis of the applicability of Ordinance 557 of the Ministry of \\ Cities in technical-economic feasibility studies for incineration \\ with MSW energy recovery in Brazil
}

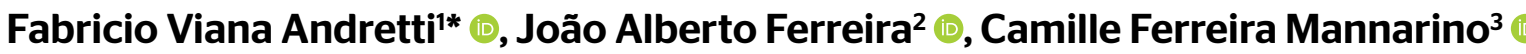

\begin{abstract}
RESUMO
Os estudos de viabilidade técnico econômica são elementos primordiais para a viabilização de projetos de saneamento, inclusive de incineração de resíduo sólido urbano com recuperação energética. O artigo teve como base os padrões para estudos de viabilidade técnico econômica conforme Portaria no 557 do Ministério das Cidades, previstos na lei no 11.445 que estabelece diretrizes nacionais para o saneamento básico. Foram realizadas visitas técnicas em quatro incineradores em operação na Suiça e dois em Portugal, com o objetivo de estabelecer um benchmarking para referenciar a análise da aplicação da Portaria, a identificação dos fatores críticos que dificultam a adoção dessa tecnologia no Brasil, assim como as ações necessárias para a viabilidade técnico-econômica do incinerador. Os principais fatores críticos identificados foram a localização da planta, o efeito Not in My Backyard, a concepção de conflito entre reciclagem e incineração, o elevado investimento inicial, o risco de adoção de tecnologias já superadas, a falta de experiência e mão de obra capacitada local, a variação e sazonalidade na alimentação de resíduo sólido urbano, a volatilidade do preço da energia no mercado, as receitas mínimas para viabilidade financeira, a adequação do arcabouço legal e a seleção de modelo de negócio adequado.
\end{abstract}

Palavras-chave: resíduos sólidos; incineração; estudo de viabilidade técnica econômica; Portaria no 557, Ministério das Cidades.

\begin{abstract}
Technical-economic feasibility studies are essential elements for making sanitation projects feasible, including projects of MSW incinerators with energy recovery. This article is based on the referencestandards for technical-economic feasibility studies according to Ordinance No. 557 (2016) of the Ministry of Cities, provided for in Law No. 11.445, which establishes national guidelines for basic sanitation. Technical visits were carried out in four incinerators in Switzerland and two in Portugal in order to establish a benchmark to reference the analysis of the application of the Ordinance, the identification of critical factors that hinder the adoption of this technology in Brazil, as well as the necessary actions for technical-economic feasibility. The objective of this article is to propose an adaptation of the technical-economic feasibility studies model of the Ordinance for a MSW incinerator project with energy recovery, identifying the critical factors and actions related to the financial-economic viability that hinder the adoption of this technology in Brazil. The critical factors identified were the location of the plant, the Not in My Backyard effect, the concept of conflict between recycling and incineration, the high initial investment, the risk of adopting technologies that have already been overcome, the lack of experience and hands of qualified local work, variation and seasonality in MSW supply, volatility in the price of energy in the market, minimum revenues for financial viability, adequacy of the legal framework, and selection of an appropriate business model.
\end{abstract}

Keywords: solid waste; incineration; techno-economic feasibility study Ordinance No. 557, Ministry of Cities.

$\square$

"Universidade do Estado do Rio de Janeiro - Rio de Janeiro (RJ). Brasil.

¿Universidade do Estado do Rio de Janeiro - Rio de Janeiro (RJ), Brasil.

3Escola Nacional de Saude Publica - Sergio Arouca Manquinhos (RJ) Brasil.

*Autor correspondente: fabricio2906engenheiro@gmail.com, fabricioandretti@tctargetconsulting.net.br

Conflitos de interesse: os autores declaram não haver conflitos de interesse.

Financiamento: bolsa de doutorado (processo E-26/201.300/2016) da Fundação de Amparo à Pesquisa do Estado do Rio de Janeiro (FAPERJ).

Recebido: 19/11/2O2O - Aceito: 11/01/2021 - Reg. ABES: 20200402 


\section{INTRODUÇÃO}

O tratamento adequado e a destinação final do resíduo sólido urbano (RSU) ainda é um grande desafio em países em desenvolvimento, onde a universalização de aterros sanitários é a prioridade. Na América Latina e Caribe, cerca de $27 \%$ das $541.000 \mathrm{t} /$ dia coletadas são dispostas de forma inadequada (UN ENVIRONMENT, 2018). No Brasil, estima-se que $40 \%$ dos resíduos coletados são dispostos de forma inadequada (SNIS, 2018; ABRELPE, 2019). Já em países desenvolvidos, o tratamento dos RSU tem padrões elevados. Na União Europeia (UE), por exemplo, das 250 milhões de toneladas anuais de RSU, 47,8\% são recicladas (incluindo compostagem e digestão anaeróbia de orgânicos), 27,4\% são tratadas em incineradores com recuperação energética e $24,8 \%$ dispostas em aterros sanitários (EUROSTAT, 2020).

São mais de 492 incineradores com recuperação energética ativos na UE, com geração potencial acima de 35 milhões de MWh de energia por ano (CEWEP, 2017). Globalmente, mais de 200 incineradores atualmente em construção estarão operacionais até 2023, a maioria em países desenvolvidos (UN ENVIRONMENT, 2019). Há um início de movimento para instalação de incineradores na América Latina (ONU MEDIO AMBIENTE, 2018), inclusive no Brasil, onde já existem quatro projetos em andamento nas regiões metropolitanas de São Paulo e Rio de Janeiro. Por isso, é fundamental que se tenha uma melhor compreensão da sua adequabilidade às condições do país e os obstáculos a serem superados para evitar projetos que consumam recursos elevados e que possam não atender os objetivos a que se destinam.

O presente artigo teve como objetivo fazer uma análise crítica sobre os padrões para realização de estudos de viabilidade técnico-econômica (EVTE) estabelecidos nas normas de referência da Portaria n 557 do Ministério das Cidades (BRASIL, 2016), e de se estabelecer um benchmarking para referenciar a análise da aplicação da Portaria e identificar as adequações e complementações e fatores críticos a serem superados e ações necessárias relacionadas à viabilidade técnico-econômica que dificultam a adoção dessa tecnologia no Brasil.

\section{METODOLOGIA}

A base do trabalho são as normas de referência, de natureza orientativa, para a elaboração de EVTE de acordo com a Portaria n 557 , do Ministério das Cidades, de 2016, previstos no art. 11, item II, da Lei no 11.445, de 2007 (BRASIL, 2016), que estabelecem diretrizes nacionais para o saneamento básico, considerando oito etapas (Figura 1).

As proposições de complementação das normas e sua adequação para EVTE para incineradores de RSU no Brasil foram estabelecidas a partir de um referencial teórico, através de pesquisa bibliográfica e do estabelecimento de benchmarking, a partir de visitas técnicas realizadas em 2017 e 2018 a quatro incineradores de RSU na Suiça e dois em Portugal. Dados de geração e tratamento de RSU em 2015 nos dois países estão na Tabela 1 e as principais características dos incineradores na Suíça e em Portugal são mostradas na Tabela 2.

As visitas às instalações dos incineradores foram seguidas de reuniões para uma discussão mais aprofundada com técnicos especializados. Também foi realizada, na Universidade do Estado do Rio de Janeiro, uma reunião com um consultor suíço sobre a problemática do uso de incineradores no Brasil.

As oito etapas da Portaria no 557 (Figura 1) foram avaliadas sequencialmente, considerando-se os principais aspectos envolvidos na utilização da incineração em sistemas de gerenciamento de RSU e analisados os fatores críticos para viabilidade financeira de um projeto de incinerador no Brasil. A etapa 8 da Portaria, de avaliação financeira, mereceu uma abordagem mais detalhada e aprofundada.

\section{RESULTADOS E DISCUSSÃO}

\section{Estudo da regionalização}

O estudo de regionalização é utilizado para definir a escala adequada para a prestação de serviços e avaliar o dimensionamento apropriado para o empreendimento, considerando a escala econômica apropriada e a possibilidade de

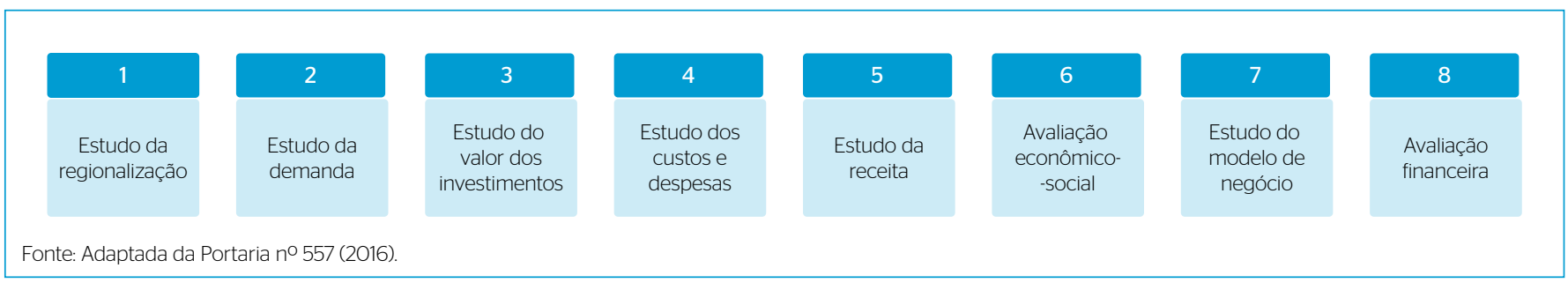

Figura 1 - Etapas para realização de estudo de viabilidade técnico-econômica.

Tabela 1 - Geração e tratamento de resíduo sólido urbano em 2015 (milhares de toneladas).

\begin{tabular}{|c|c|c|c|c|c|}
\hline & Gerada & Compostagem & Reciclagem & Incineração & Aterro \\
\hline Portugal & 4.710 & 665 & 765 & 974 & 2.307 \\
\hline Suiça & 6.030 & 1.256 & 1.924 & 2.850 & 0 \\
\hline
\end{tabular}

Fonte: elaborada pelos autores com base em Eurostat (2016). 
Tabela 2 - Características dos incineradores nas visitas técnicas realizadas na Europa.

\begin{tabular}{|c|c|c|c|c|c|c|}
\hline País & Suíça & Suíça & Suiça & Suiça & Portugal & Portugal \\
\hline Nome local da Planta & $\begin{array}{c}\text { SIG Les } \\
\text { Cheneviers }\end{array}$ & Centre TRIDEL & KVA Oftringen & KVA Hagenholz & LIPOR & VALORSUL \\
\hline Inicialização' & 1993 & 2006 & 1992 & 2010 & 1999 & 2004 \\
\hline Quantidade de RSU tratada na planta (t/dia) & 696 & 468 & 192 & 720 & 1.280 & 2.000 \\
\hline Quantidade de RSU tratada na planta (t/ano) & 231.799 & 156.000 & 64.000 & 240.000 & 441.600 & 662.000 \\
\hline Geração energia elétrica anual (MWh) & 106.000 & 60.000 & 54.000 & 338.000 & 197.000 & 300.000 \\
\hline Geração térmica anual (MWh) & 250.000 & 260.000 & 160.000 & 250.000 & - & - \\
\hline Norma de controle de emissões & \multicolumn{4}{|c|}{ Ordonnance sur la protection de l'air (OPair) (OFEV, 2018) } & \multicolumn{2}{|c|}{ Diretiva 2010/75/CE (UE, 2010) } \\
\hline Número de linhas & 3 & 2 & 1 & 2 & 2 & 3 \\
\hline Poder Calorífico Nominal (MJ/kg) & $\begin{array}{c}\text { 13,1 (L1) } \\
11,6(\mathrm{~L} 2, \mathrm{~L} 3)\end{array}$ & 14,4 & 11,6 & $\begin{array}{l}11,3(\mathrm{~L} 1) \\
11,7(\mathrm{~L} 2)\end{array}$ & 7,7 & 7,8 \\
\hline Forno (TIPO) & \multicolumn{6}{|c|}{ Grelha Móvel } \\
\hline Modal de recebimento & $\mathrm{RO}+\mathrm{FL}$ & $\mathrm{RO}+\mathrm{FE}$ & $\mathrm{RO}$ & $\mathrm{RO}$ & $\mathrm{RO}$ & $\mathrm{RO}$ \\
\hline Tarifa $(€ / t)^{2}$ & 100 & 190 & 100 & 100 & 20 & 24 \\
\hline CAPEX $(\text { US } \$ / t)^{3}$ & \multicolumn{4}{|c|}{ Valores não fornecidos } & 435 & 423 \\
\hline
\end{tabular}

': nova linha ou atualização; ${ }^{2}$ : gate fee cobrado para recepção do RSU bruto; ${ }^{3}$ : valores atualizados para 2O16; RO: rodoviário; FL: fluvial; FE: ferroviário; RSU: resíduo sólido urbano. Fonte: Próprio autor (visitas técnicas 2017 e 2018).

consorciamento entre municípios (BRASIL, 2016). A Tabela 3 mostra os principais fatores a serem considerados na localização de um incinerador de RSU.

A proximidade de polos industriais e de subestação de energia elétrica é bastante relevante para reduzir perdas por dissipação da energia a ser comercializada, que tem peso significativo na receita de incineradores de RSU.

As questões relativas ao acesso a área podem ser importantes no planejamento do transporte dos resíduos. Entre as plantas visitadas na Suíça, duas operam com outros modais além do rodoviário, o SIG (fluvial) e Tridel (ferroviário). Embora seja comum encontrar regulamentos rígidos para localizar instalações de gerenciamento de RSU em áreas densamente povoadas, na UE, diversas plantas estão localizadas no centro de cidades como Paris, Viena, Copenhague etc. (COELHO, 2020). Na China, a maioria das plantas estão localizadas nos centros urbanos mais desenvolvidos economicamente (ZENG et al., 2013).

Outro fator crítico pode ser a rejeição da comunidade com base em argumentos como distúrbios causados pelo aumento do tráfego, principalmente à noite, os odores, e o mais importante, a percepção de que a saúde estará em risco (COELHO, 2020).

\section{Estudo da demanda}

A expectativa de demanda pelos serviços deve ser referenciada em estudos populacionais para um horizonte de trinta anos com projeções de quantitativos de resíduos coletados e população a ser atendida para cada período de contrato, incluindo também análises de sensibilidade dos mesmos (BRASIL, 2016). No caso do incinerador, além da demanda por tratamento de RSU, deve-se considerar a demanda por energia elétrica e/ou térmica. Segundo o CEWEP (2017), a recuperação total de energia de um incinerador moderno é de 50 a $70 \%$ do conteúdo energético dos RSU, dos quais 15 a 25\% são energia elétrica e o restante é energia térmica, com a proporção variando de acordo com as demandas locais. Nas plantas de Portugal, é feita essencialmente a conversão elétrica enquanto na Suíça são recuperadas energia elétrica e principalmente térmica.
Tabela 3 - Fatores a serem considerados na localização de incineradores.

\begin{tabular}{l|c} 
Principais Fatores & Justificativas \\
$\begin{array}{l}\text { Proximidade do centro de } \\
\text { massa gerador de RSU }\end{array}$ & Custos de transporte \\
\hline $\begin{array}{l}\text { Proximidade de polos } \\
\text { industriais }\end{array}$ & Comercialização de energia térmica \\
\hline Acesso ao sistema elétrico & Comercialização de energia elétrica \\
\hline Acesso à área & Vias de acesso, fluxo rodoviário e trânsito local \\
\hline Legislação local & Restrições no zoneamento da cidade \\
\hline Custo da área & Valor que compõe o investimento inicial \\
\hline Local de disposição da escória & Disponibilidade de aterro sanitário \\
\hline $\begin{array}{l}\text { Proximidade de áreas } \\
\text { residenciais }\end{array}$ & Rejeição da comunidade
\end{tabular}

RSU: resíduo sólido urbano.

Fonte: elaborada pelos autores com base em Chirico (1996), GIZ (2017) e Coelho (2O2O).

Segundo diversos autores, os valores de geração de energia nas plantas atuais podem variar entre 400 e $700 \mathrm{KWh}$ por tonelada de RSU nos países desenvolvidos e de 300 a $400 \mathrm{KWh}$ nos em desenvolvimento, em função de diferenças do poder calorífico dos resíduos (MARTIN et al., 2017; YAN et al., 2020). Nos dados de benchmarking levantados em Portugal e na Suíça fica claro a maior recuperação energética neste último, onde o poder calorífico dos resíduos tratados é cerca de 55\% maior (de 12 para 7,7 MJ/kg).

É possível que a taxa de reciclagem de materiais secos possa interferir no poder calorífico inferior (PCI) dos resíduos a serem incinerados e dar margem a uma discussão sobre conflito entre a reciclagem e o uso de energia dos RSU, podendo ser um fator para o não uso da incineração em vários países 
(CUCCHIELLA et al., 2012; GIZ, 2017). Os países da UE que mais utilizam incineradores de RSU apresentam também elevados índices de reciclagem, demonstrando que a tecnologia de incineração é compatível com a reciclagem (EUROSTAT, 2016). Os dados da Tabela 1 mostram que em Portugal, onde são incinerados $21 \%$ dos RSU gerados, a taxa de reciclagem é de $16 \%$, e na Suíça, que incinera $47 \%$ do total, ela atinge um padrão de 32\% dos RSU. No Brasil, é importante considerar a prioridade da reciclagem antes dos tratamentos, conforme já estabelecida na Política Nacional de Resíduos Sólidos (PNRS).

Embora a preocupação com os postos de trabalho de trabalhadores que atuam na catação e triagem de materiais recicláveis em muitos municípios brasileiros mereça sempre ser considerada (COELHO, 2020), os índices de reciclagem (resíduos secos) de 7\% no país (SNIS, 2018) indicam uma margem muito grande de resíduos que poderiam ser considerados para projetos de incineração sem que ocorram impactos significativos para os "catadores".

Em países onde há metas estabelecidas de desenvolvimento sustentável, a incineração de RSU com recuperação energética contribui para melhorar o padrão da geração de energia renovável e para a redução de impactos ambientais (IEA, 2013; BRUNNER \& RECHBERGER, 2015).

\section{Estudo de valor do investimento}

O CAPital EXpenditure (CAPEX) dos incineradores são as despesas de capital ou investimentos, e referem-se ao somatório dos custos relacionados ao planejamento e construção do projeto (CAPEX $=\mathrm{C}=\sum_{i=1}^{n} C i$ ). O CAPEX dos equipamentos é influenciado pelo tipo de energia que se pretende recuperar (MAISIRI et al., 2015). Com base nos valores médios obtidos nas visitas técnicas, do CAPEX total, os equipamentos de processamento térmico (incinerador/caldeira) $\left(C_{1}\right)$ representam $35 \%$, os equipamentos de produção de energia (turbinas e geradores) $\left(\mathrm{C}_{2}\right)$ representam $10 \%$, o sistema de tratamento de gases de combustão $\left(\mathrm{C}_{3}\right)$ representam $25 \%$, as obras civis $\left(\mathrm{C}_{4}\right)$ representam $20 \%$ e os demais elementos (aquisição do terreno, documentação, equipamentos para processamento de cinzas, transmissão elétrica etc.) $\left(\mathrm{C}_{5}\right)$ representam $10 \%$. Também devem ser quantificados os custos das medidas mitigadoras e compensatórias de caráter social e ambiental que, opcionalmente, ao invés de fazer parte do CAPEX, podem estar incluídos nos custos e despesas (BRASIL, 2016).

As melhores práticas de incineração recomendadas pela European Commission (2019) incluem padrões sobre o uso da tecnologia, o projeto e construção da planta, a manutenção/ operação e desativação, e o controle dos padrões de emissões atmosféricas, efluentes e resíduos do processo. A combustão em grelha, utilizada nos seis incineradores visitados, é uma tecnologia comercialmente comprovada em uso há mais de 130 anos e a mais utilizada mundialmente em relação a outras tecnologias de processamento térmico de RSU (IEA, 2013; CHALIKI et al., 2016; MARTIN et al., 2017). Na UE, 90\% das plantas usam incineração de grelha assim como mais de 800 instalações em todo o mundo (IEA, 2013).

Com base nos dados de 2013, corrigidos anualmente, o CAPEX dos incineradores na Europa está na faixa de US\$ 710 a 1.065 por tonelada da capacidade anual, com um valor médio da ordem de US\$ 880/t (cerca de $\mathrm{R} \$ 4.500,00 / \mathrm{t}$ ) (CEWEP, 2011; O’BRIEN, 2013; THEMELIS et al., 2013). Nas visitas técnicas, não foi possível obter os valores de CAPEX dos incineradores da Suíça, mas o projeto da planta de Lucerna/Perlen (Suíça) está acima de US\$1.000/t da capacidade anual (HZI, 2016). Conforme pode ser visto na Tabela 2, os valores de CAPEX dos incineradores de Portugal tem valores de US\$ 435/t (Lipor) e US\$ 423/t (Valorsul) (ABRANTES, 2016), quase 50\% menores do que a média da UE. Isto se explica em função de uma política de estado da UE com investimentos a fundo perdido para os países membros menos desenvolvidos para os setores de infraestrutura e serviços (inclusive na gestão de RSU), com o objetivo de reduzir as desigualdades na região como um todo. De acordo com a CRI (2014), o CAPEX médio de incineração com recuperação de energia na China é de US\$228/t, consideravelmente menor do que os valores da UE. Na China, além da mão de obra ser mais barata quando comparada com a Europa e o Brasil, as empresas de rede elétrica são obrigadas a comprar a eletricidade gerada pelos incineradores de RSU a um preço diferenciado subsidiado do governo (CRI, 2014).

Os elevados valores iniciais de CAPEX podem representar um fator crítico para a implementação de incineradores nos países em desenvolvimento. Há que se estar atento a projetos de menor CAPEX que podem implicar em padrões de emissões inferiores com tecnologias superadas (DORN et al., 2012). Os projetos de baixo CAPEX podem, por exemplo, não empregar sistemas adequados de tratamento de gases de combustão, utilizar aço e refratários de baixa qualidade nos componentes da planta (ex.: forno), além de aumentarem o risco de quebras não planejadas, ou aumento da corrosão de componentes críticos, levando, com isso, a uma vida útil operacional mais curta da planta (GIZ, 2017). Em consequência, pode haver um aumento dos custos de operação e manutenção, além de uma possível redução da taxa de utilização da operação da planta, impactando o fluxo de entrada das receitas.

A redução da taxa de juros de financiamento a ser obtida é um fator crítico para viabilização financeira de projeto de incinerador. Os custos e investimentos ao longo do tempo trazidos a valor presente são diretamente influenciados pela taxa de juros bancária adquirida para o financiamento. Portanto, a viabilidade econômica de um projeto de grande aporte de capital, como o incinerador, depende diretamente do nível de taxa de juros obtida.

\section{Estudo dos custos e despesas}

O OPerational EXpenditure (OPEX) são os gastos no horizonte da vida útil do empreendimento (em geral 30 anos para incineradores) contemplando estimativas dos principais componentes dos custos operacionais e despesas administrativas, pressupondo a prestação do serviço em regime de eficiência (BRASIL, 2016). As estimativas do OPEX devem ser fundamentadas com premissas, de preferência com uso de referências mundiais e/ou por meio de cotações atualizadas de fornecedores disponíveis no mercado. Os custos tributários devem ser discriminados e obedecer à legislação vigente local (BRASIL, 2016). A estimativa do OPEX total (OPEX $=\mathrm{O}=\sum_{i=1}^{n} \mathrm{O}$ ) tem como principais elementos: operação e manutenção $\left(\mathrm{O}_{1}\right)$, mão de obra (própria e terceiros) $\left(\mathrm{O}_{2}\right)$, combustíveis $\left(\mathrm{O}_{3}\right)$, insumos $\left(\mathrm{O}_{4}\right)$, logística de destinação dos rejeitos $\left(\mathrm{O}_{5}\right)$, seguros $\left(\mathrm{O}_{6}\right)$, despesas gerais administrativas $(\mathrm{DGA})\left(\mathrm{O}_{7}\right)$, impostos (custo tributário) $\left(\mathrm{O}_{8}\right)$ (EHRHARDT \& BRIGHAM, 2010), e provisionamento do custo de desmobilização $\left(\mathrm{O}_{9}\right)$. Comparado com outras tecnologias de tratamento de RSU, o incinerador envolve um grande investimento de capital (CAPEX) e também elevado OPEX (ZHANG et al., 2015), o que é um fator crítico para a sustentabilidade financeira do projeto.

A característica térmica dos RSU é um fator crítico na indústria da incineração e tem que ser analisado antes da escolha da tecnologia (COELHO, 2020). Se a composição dos RSU de entrada for diferente daquela para a qual a planta foi projetada (por exemplo, menor PCI), os componentes da planta podem 
degradar mais rápido e pode haver dificuldades para atender aos padrões rigorosos de emissões, além da necessidade de gastos com combustíveis auxiliares (GIZ, 2017). As características também estão sujeitas a variações sazonais, que podem afetar a incinerabilidade dos RSU e devem ser analisadas previamente. Nos incineradores de Lipor e Valorsul é requerido, com frequência, o uso de combustível auxiliar, uma vez que o PCI do RSU é baixo.

O número médio de empregos diretos criados por uma planta de incineração na Europa é de 62 (CHALIKI et al., 2016). Dos incineradores visitados, os incineradores de Lipor e Valorsul tem 45 e 71 funcionários respectivamente, enquanto na Suíça o número médio de funcionários é inferior a 50. A falta de experiência e mão de obra capacitada local para a operação da planta irão adicionar um desafio extra (COELHO, 2020).

\section{Estudo das receitas}

A análise das receitas deve considerar o ciclo de vida útil do projeto (30 anos) e deve contemplar a quantificação das receitas emergentes da prestação dos serviços (BRASIL, 2016). A receita total (Receita $=\mathrm{R}=\sum_{i=1}^{n} R i$ ) corresponde aos acréscimos de benefícios econômicos relativos às operações (FAS, 2002). As principais fontes de receita para um incinerador são a tarifa recebida por tonelada de RSU tratado $\left(R_{1}\right)$, a venda de eletricidade $\left(R_{2}\right)$ e/ou energia térmica $\left(R_{3}\right)$, a venda de metais (escória) $\left(R_{4}\right)$, e a comercialização das cinzas residuais para fins de construção civil $\left(\mathrm{R}_{5}\right)$, sendo esta alternativa dependente de legislação local. Além disso, podem ocorrer potenciais receitas adicionais dos créditos de carbono gerados $\left(\mathrm{R}_{6}\right)$. Mas como essas receitas, por si só, podem não ser suficientes para a viabilidade financeira, podem ser então necessárias taxas adicionais ou subsídios governamentais para cobrir os custos (GIZ, 2017).

A redução da volatilidade do preço de venda de energia e a adequação da tarifa recebida por tonelada de RSU tratada são fatores críticos para a viabilidade econômica. A volatilidade do preço da energia elétrica no mercado é consequência do modelo de comercialização (no caso do Brasil é através de leilões regulados periódicos) e impacta diretamente a projeção de receitas para a viabilidade econômica, podendo ser mitigada por meio de contratos de fornecimento de energia de longo prazo, como no caso dos incineradores de Portugal. Alguns países assimilam RSU a um recurso renovável e qualificam o incinerador nos subsídios à energia verde como Portugal (COELHO, 2020). Os incineradores Valorsul e Lipor possuem subsídio de tarifa especial "verde" da eletricidade produzida no valor de 85 euros/MWh. Na China, a rede elétrica é obrigada a buscar eletricidade gerada pelo incinerador a um preço especial que inclui um subsídio (CRI, 2014). O Reino Unido, a Itália e a Espanha, entre outros, apoiaram a incineração por meio de preços elevados para a eletricidade gerada nas plantas.

Em relação à tarifa recebida por tonelada de resíduo tratado, o valor mínimo para a viabilidade financeira do incinerador pode ser superior ao praticado para disposição dos RSU em aterros em operação no país. Nos EUA os valores médios das tarifas para incineração de RSU são da ordem de US\$ 60-110/t (EREF, 2017), no Reino Unido de 104 US\$/t (WRAP, 2018) e na Suécia é de US\$ 84/t (WORLD ENERGY COUNCIL, 2016). Nos incineradores da Suíça, as tarifas são acima de US\$100/t. Em Portugal, as tarifas estão entre 27-40 US\$/t, inferior à média da UE, em função do país possuir uma média salarial inferior; de não haver imposto sobre resíduos ou aterros; além de grande parte do financiamento para infraestrutura ter sido financiado pela UE ou fornecidas baixas taxas de juros pelo banco europeu de investimento.

\section{Avaliação econômico-social}

Em projetos com impacto direto em mais de cem mil pessoas, recomenda-se a elaboração de estudo econômico-social que contemple os aspectos relacionados à identificação de benefícios e custos econômicos, à estimativa das externalidades positivas e negativas do projeto, e à identificação do custo de oportunidade do poder público, por meio da taxa de desconto social ou outro método tecnicamente justificado (BRASIL, 2016). A tecnologia de incineração de quarta geração, vigente hoje e utilizada nos seis incineradores visitados, emprega uma série de equipamentos responsáveis pela redução da emissão e remoção de outros poluentes como os óxidos de nitrogênio, dioxinas e furanos, com controle ambiental marcado por legislações mais restritivas (NZIHOU et al., 2012).

Na UE, a diretiva 2010/75/CE (Prevenção e Controle Integrado da Poluição - IPPC) visa reduzir as emissões no ar, solo, água e terra e prevenir a geração de resíduos, a fim de alcançar um alto nível de proteção do meio ambiente, estabelecendo limites de emissão específicos (UE, 2019). A legislação Suíça (OFEV, 2018) e a diretiva 2010/75/CE da UE, aplicadas aos incineradores visitados, são dos padrões mais rigorosos para a incineração de RSU no mundo, com padrões difíceis de serem atendidos pelos países em desenvolvimento (YAN et al., 2020).

A incineração de resíduos no Brasil está sujeita às disposições da resolução CONAMA n ${ }^{\circ} 316$, que regulamenta os métodos de tratamento térmico de resíduos e estabelece os limites dos parâmetros de emissões (BRASIL, 2002) menos rigorosos que a UE. Por outro lado, a resolução $\mathrm{n}^{\circ} 079$ da Secretaria do Meio Ambiente do Estado de São Paulo (SMA/SP) estabelece os limites dos parâmetros para o controle da emissão de poluentes em plantas de processamento térmico de RSU (SMA, 2009) tão restritivas quanto as preconizadas pela UE.

O incinerador da Tridel (Suíça) obteve valores médios dos parâmetros (ácido clorídrico, ácido fluorídrico, dióxido de enxofre, nitrogênio amoniacal, mercúrio, cádmio, chumbo + zinco e monóxido de carbono) no mínimo 88\% abaixo dos limites estabelecidos pelas legislações Suíça e europeia nos anos de 2017, 2018 e 2019 (TRIDEL, 2019). A legislação ambiental, em países em desenvolvimento, tem padrões de exigências, em certos parâmetros, inferiores aos da UE, como por exemplo: as partículas inaláveis $\mathrm{PM}_{10} 24 \mathrm{~h}\left(\mathrm{em} \mu \mathrm{g} / \mathrm{m}^{3}\right)$ :

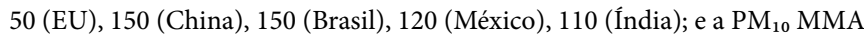
(Média Anual Aritmética) (em $\mu \mathrm{g} / \mathrm{m}^{3}$ ): 40 (UE), 70 (China), 50 (Brasil), 50 (México), 60 (Î́ndia).

No tocante à questão social, a implementação de incineradores nos países em desenvolvimento apresenta desafios como os relacionados à aceitação por parte da população local, que desconhece a tecnologia e a vê como uma fonte de poluição (VERGARA \& TCHOBANOGLOUS, 2012). Com isso, a adequação do arcabouço legal se torna um fator crítico, sendo a implementação de uma legislação nacional tão restritiva quanto as estabelecidas pela UE, um pré-requisito para a introdução dos incineradores nos países em desenvolvimento (UN ENVIRONMENT, 2019). A legislação deve cumprir os limites de emissões reconhecidos internacionalmente para evitar danos ao meio ambiente e à saúde pública (GIZ, 2017). Para isto, um importante desafio é o apoio político, pois é a base para qualquer avanço no gerenciamento de resíduos (RAGOSSNIG \& VUJIÉ, 2015).

\section{Estudo do modelo de negócio}

A Portaria $n^{\circ} 557$ recomenda que o estudo do modelo de negócio contemple a análise das alternativas e a avaliação dos diversos modelos contratuais, incluindo a justificativa do modelo contratual escolhido e demonstrando suas vantagens 
sociais (BRASIL, 2016). Com as restrições no investimento público nos países em desenvolvimento, cada vez mais as Parcerias Público Privadas (PPP) vêm sendo instrumentos de viabilização de projetos de saneamento básico. Um modelo de negócios adequado é essencial para atrair investidores privados, em especial, nos países em desenvolvimento que possuem limitações orçamentárias para o investimento público. Diferentes modelos de negócios estão em vigor em diferentes países e cidades. A incineração na UE começou praticamente no setor público, com a exceção da França, entretanto, pode ser detectada uma tendência de amplo envolvimento do setor privado especialmente no campo da energia (MASSARUTTO, 2007). Na China, não houve participação pública quando as plantas de incineração foram desenvolvidas inicialmente (ZHANG et al., 2015). Todos os incineradores visitados na Suíça são empresas públicas e os de Portugal são atualmente de empresas privadas.

PPPs têm diferentes características nos modelos de negócios em função das especificidades de cada país e dependem do orçamento financeiro e do nível em que o poder público está disposto e seja capaz de subsidiar o incinerador para assegurar a viabilidade a longo prazo (YAN et al., 2020). Existe uma lacuna na literatura internacional sobre evidências de diferença de desempenho entre sistemas operados por serviços públicos e privados e qual seria mais apropriado para situações específicas (SOÓS et al., 2017). Provavelmente, uma combinação de prestadores de serviços públicos e privados pode contribuir para uma estrutura mais resiliente para soluções de gestão sustentável e o sucesso do projeto deve atender aos interesses da sociedade e de ambos os setores (PASIN et al., 2003). Nos Emirados Árabes Unidos, o projeto de duas plantas de incineração, num total de 1.500.000 t/ano de capacidade com geração de $150 \mathrm{MW}$ de eletricidade, será executado através de PPP (ECOPROG, 2020).

Uma das vantagens das PPPs é que elas viabilizam projetos financeiramente, sendo os riscos de conclusão e resultados compartilhados com o governo e o operador/investidor privado (UN ENVIRONMENT, 2019). Utilizando essas parcerias, o governo continua atuando de acordo com o contrato de concessão administrativa na modalidade patrocinada ou administrativa (CARVALHO FILHO, 2016). Apesar disso, apenas $15 \%$ das concessões e projetos de PPP propostos no Brasil terminam na assinatura dos contratos (FIRJAN, 2019).

\section{Avaliação financeira}

Embora a Portaria ${ }^{\circ} 557$ apresente os principais elementos recomendados para a avaliação financeira (etapa 8 da Figura 1), existem lacunas que exigem uma melhor orientação. Assim, é apresentada uma proposta de itens complementares para a realização da análise financeira, elencados na Figura 2 e discutidos na sequência.

\section{Parâmetros operacionais}

Para estabelecer os fatores básicos considerados no estudo do valor do investimento é necessário, preliminarmente, realizar uma avaliação da capacidade e desempenho do projeto do incinerador. Os principais parâmetros operacionais e financeiros de projeto são: (a) capacidade da planta (t/ano); (b) número de linhas (qtd); (c) vida útil da planta (anos); (d) período de construção (anos); (e) capacidade de processamento da planta por linha (t/ano/linha); (f) disponibilidade da planta (\%); (g) PCI médio dos resíduos (GJ/t); (g) eficiência da geração de eletricidade (\%); (h) eficiência da geração de vapor (\%); capacidade de projeto da planta (MW); (i) potência total de saída $(\mathrm{kWh} / \mathrm{t}) ;(\mathrm{j})$ consumo de planta $(\mathrm{kWh} / \mathrm{t}) ;(\mathrm{l})$ eletricidade para venda na rede $(\mathrm{kWh} / \mathrm{t}) ; \mathrm{e}(\mathrm{m})$ mão de obra total (qtd própria + terceira).

\section{Premissas financeiras}

As premissas básicas de entrada para um modelo financeiro das plantas de incineração devem ser estabelecidas e utilizadas no cenário base na análise financeira, são elas: (a) custo de capital (CAPEX) por tonelada (\$/t); (b) custo de manutenção (\% do custo do capital); (c) custo por tonelada tratada (\$/t); (d) preço de venda da eletricidade ( $\$ / k W h) ;(e)$ preço de venda de metais ferrosos $(\$ / k g)$; (f) preço de venda de metais não ferrosos (\$/kg); (g) taxa de depreciação (\%); (h) taxa de desconto (taxa de longo prazo) (\%); (i) projeção de inflação (\%); (j) taxa de juro (\%); (l) impostos (\%); (m) percentual do CAPEX financiado por uma instituição financeira (\%); (n) custo médio do trabalho (\$/pessoa/ano); (o) despesas gerais administrativas; (p) custo de produtos químicos $(\$ / \mathrm{kg} / \mathrm{t})$ e; custo da água $(\$ / \mathrm{L} / \mathrm{t})$.

\section{Elaboração da Demonstração de Resultados de Exercício}

São calculados considerando o fluxo de caixa dos investimentos, os custos (fixos e variáveis), as despesas e as receitas, estruturadas em uma Elaboração da Demonstração de Resultados de Exercício (DRE) ${ }^{1}$ para 30 anos. O custo do ciclo de vida de um ativo pode ser significativamente superior ao valor de investimento inicial, e deve ser avaliado através da DRE logo na fase de projeto (BESCHERER, 2005). A estruturação da DRE deve incluir as divisões relacionadas ao modelo de negócio adotado segregando as responsabilidades financeiras e fiscais de entidades público e privadas.

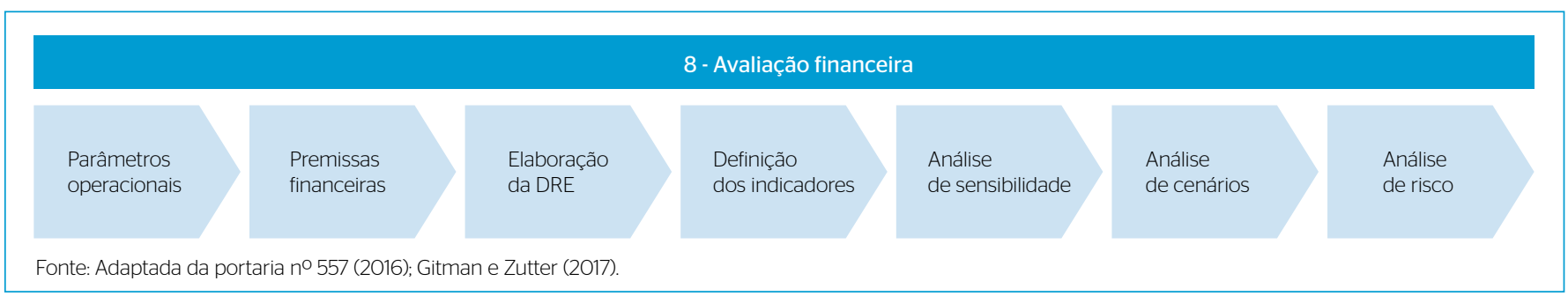

Figura 2 - Itens para realização da análise financeira.

${ }^{1}$ Instituído no artigo 187 da Lei 6.404/1976 (Lei das Sociedades por Ações). No atual Código Civil Brasileiro, a DRE corresponde ao “resultado econômico”, cujo levantamento é obrigatório conforme seu artigo 1.179 


\section{Definição dos indicadores}

Como base para análises, o estudo financeiro pode focar, minimamente, mas não exclusivamente, nos indicadores tradicionais específicos para avaliação da viabilidade financeira, a saber: Valor Presente Líquido (VPL), Taxa Interna de Retorno (TIR) e retorno do investimento (Return Of Investiment - ROI) (ROSS et al., 2018; GITMAN \& ZUTTER, 2017), apresentados na Tabela 4.

O VPL de um projeto é definido como a soma algébrica dos valores descontados do fluxo de caixa a ele associado. A TIR é a taxa de retorno exigida que, quando usada como taxa de desconto, resulta em um VPL igual a zero (FILHO et al., 2016; LEMES et al., 2016; ROSS et al., 2018). O ROI mede a quantidade financeira de retorno de um investimento, em relação ao custo do investimento (GITMAN \& ZUTTER, 2017). O VPL e a TIR são nomeados em grande parte da literatura financeira como os métodos mais tradicionais na avaliação de projetos de investimentos (SCHROEDER et al., 2005).

\section{Análise de sensibilidade}

A análise de sensibilidade procura estimar o resultado final de acordo com oscilações das variáveis determinantes. Em um estudo de pré-viabilidade, pode-se utilizar um grau de "imprecisão" de até + /- 20\% como base do orçamento de investimento (UNIDO, 2019). Uma variação do investimento inicial de $\pm 20 \%$ pode ter um impacto de $153 \%$ a $+105 \%$ no resultado financeiro do projeto (MASSARUTTO, 2015). A análise de sensibilidade de VPL, TIR e ROI pode ser realizada considerando variação nas duas principais variáveis de receita (preço de eletricidade e a tarifa por tonelada) que mais afetam o desempenho financeiro do projeto (em alguns países pode ser considerado também o preço da energia térmica). Uma escala de atratividade de investimento deve ser formulada para análise dos resultados de sensibilidade de VPL, TIR e ROI, podendo adotar as faixas indicadas no Quadro 1.

\section{Análise de cenários}

Além da análise de sensibilidade, devem ser calculados resultados financeiros para diferentes cenários. O exercício de planejar por cenários ajuda a identificar possíveis estratégias (VAN DER HEIJDEN, 2009). Em função do nível de significância dentre as diversas variáveis de um modelo financeiro do incinerador, pelo menos dois cenários devem ser considerados na análise, envolvendo duas variáveis de maior efeito/impacto no resultado financeiro, das quais são indicadas: (a) a variação da taxa de juros do banco e (b) o nível de CAPEX. A taxa de juros pode ser definida como o preço do uso do dinheiro ao longo de um determinado período de tempo (BAUMANN, 2004). O primeiro cenário deve considerar uma taxa de juros bancária inferior à aplicada no cenário base para o caso de obtenção de linhas

Tabela 4 - Principais indicadores tradicionais específicos para avaliação financeira.

\begin{tabular}{l|c} 
Indicadores financeiros & Fórmula \\
\hline Valor presente líquido (em \$) & $V P L=-\mathrm{I}+\sum_{t=1}^{n} \frac{F C_{\mathrm{t}}}{(1+i)^{t}}$ \\
\hline Taxa interna de retorno (em \%) & $\sum_{t=1}^{n} \frac{F C_{\mathrm{t}}}{(1+T I R)^{t}}=0$ \\
\hline Retorno do investimento (em \%) & ROI $=\frac{(\text { Receita-custo) }}{\text { Custo }} x 100=\left(\frac{\left.\sum_{i=1}^{n} R i-\sum_{i=1}^{n} C i-\sum_{i=1}^{n} 0 i\right)}{\left(\sum_{i=1}^{n} C i+\sum_{i=1}^{n} 0 i\right.}\right) x 100$
\end{tabular}

FC: fluxo de caixa; l: investimento inicial; R: receita; C: CAPEX; O: OPEX; t: tempo. Fonte: elaborada pelos autores com base em Ross et al. (2018); Lemes et al. (2016); Gitman e Zutter (2017). de financiamento junto aos bancos nacionais ou internacionais e no caso de adoção de algum tipo de incentivo público pela redução de imposto de importação. O segundo cenário considera uma redução no CAPEX para o caso de adoção de algum tipo de incentivo pelo poder público em termos de imposto de importação e/ou adoção de tecnologias de baixo custo frente as referências mundiais, como é o caso da China (CRI, 2014). Ambos os cenários devem ser comparados ao cenário base e ranqueados de acordo com o desempenho de VPL, TIR e ROI.

\section{Análise de risco}

Os riscos constituem o conjunto de eventos capazes de comprometer o alcance dos objetivos de uma organização ou empreendimento (COSO, 2017; ISO, 2018), e podem ser analisados a partir de uma faixa de variação associada a cada elemento do fluxo contábil, bem como de seus prazos (GITMAN e ZUTTER, 2017). A identificação dos riscos se baseia na oportunidade e na ocorrência do evento versus o impacto do mesmo (WALKER, 2013; AQLAN \& ALI, 2014) e através da abordagem de gerenciamento (CHOI et al., 2016) e monitoramento dos riscos (WALTERS, 2007). Para os projetos de incineradores, pode-se utilizar como base a quantificação dos riscos associados aos fatores críticos identificados e sintetizados no Quadro 2, buscando compreender as causas, suas implicações e ações propostas.

\section{Resumo dos fatores críticos e ações propostas}

O Quadro 2 apresenta um resumo dos principais fatores críticos e ações propostas relacionados à viabilidade financeira-econômica na implementação de incineradores de RSU com recuperação energética no Brasil.

\section{CONCLUSÃO}

$\mathrm{O}$ uso de incineradores em países em desenvolvimento, como o Brasil, pode contribuir para a gestão de RSU, particularmente em grandes cidades onde haja maiores dificuldades para ampliação do uso de aterros sanitários. Entretanto, sua implementação ainda apresenta desafios a serem enfrentados como: a superação da rejeição das comunidades a partir da determinação de todas as externalidades positivas e negativas e, de comum acordo, o estabelecimento de medidas mitigadoras e compensatórias; a confirmação do PCI dos resíduos incinerados ao longo da vida útil do incinerador, alinhada com as estratégias do Plano

Quadro 1 - Escala de atratividade proposta para análise de sensibilidade.

\begin{tabular}{|c|c|}
\hline Indicadores & Critérios \\
\hline \multirow{4}{*}{ VPL } & VPL $\leq$ O: investimento não financeiramente viável \\
\hline & $\mathrm{O}<\mathrm{VPL} \leq 1 / 4$ do custo capital: viável mas não atraente \\
\hline & $1 / 4<$ VPL $\leq 1 / 2$ do custo de capital: viável e atraente \\
\hline & $1 / 2$ do custo de capital < VPL: viável e mais atraente \\
\hline \multirow{3}{*}{ TIR } & TIR $\leq 10 \%$ : investimento não financeiramente viável \\
\hline & 10\% < TIR $\leq 15 \%$ : viável mas não atraente \\
\hline & TIR > 15\%: viável e mais atraente \\
\hline \multirow{3}{*}{$\mathrm{ROI}$} & ROI $\leq$ O\%: investimento não financeiramente viável \\
\hline & O\% < ROI $\leq 5 \%$ : viável mas não atraente \\
\hline & ROI > 5\%: viável e mais atraente \\
\hline
\end{tabular}

VPL: valor presente líquido; TIR: taxa interna de retorno; ROI: retorno de investimento. Fonte: Próprio autor. 
Quadro 2 - Fatores críticos e ações propostas.

\begin{tabular}{|c|c|c|}
\hline Portaria 557 & Fatores críticos & Ações propostas \\
\hline \multirow{2}{*}{$\begin{array}{l}\text { Estudo da } \\
\text { regionalização }\end{array}$} & Localização da planta & $\begin{array}{c}\text { Dada a existência de multivariáveis, aplicar modelo matemático de otimização logística usando software de } \\
\text { programação linear e/ou sistema de informação geográfica }\end{array}$ \\
\hline & Efeito not in my backyard (NIMBY) & $\begin{array}{l}\text { Estabelecer plano de comunicação com todas as partes interessadas (autoridades, setor de resíduos, setor de } \\
\text { energia e comunidade) para garantia na participação do processo desde a concepção do projeto }\end{array}$ \\
\hline \multirow[b]{2}{*}{$\begin{array}{l}\text { Estudo da } \\
\text { demanda }\end{array}$} & \multirow[b]{2}{*}{$\begin{array}{l}\text { Concepção de conflito entre } \\
\text { reciclagem e incineração }\end{array}$} & Aplicar a incineração em grandes centros urbanos desenvolvidos economicamente \\
\hline & & $\begin{array}{c}\text { Considerar a prioridade da reciclagem antes dos tratamento conforme estabelecida na PNRS, o que implica que } \\
\text { catadores continuarão a ser necessários na implementação e ampliação da coleta seletiva nos municípios, o que } \\
\text { deverá ser considerado nos projetos de incineradores }\end{array}$ \\
\hline \multirow{5}{*}{$\begin{array}{l}\text { Estudo do valor } \\
\text { dos investimentos }\end{array}$} & \multirow{3}{*}{ Elevado capex inicial } & $\begin{array}{l}\text { Estabelecer linhas especificas de financiamento com ofertas de taxas de juros diferenciadas nos bancos de } \\
\text { desenvolvimento, para conseguirem atrair investidores privados e reduzir as despesas com o custo de capital }\end{array}$ \\
\hline & & Avanço tecnológicos na redução dos preços das tecnologias por parte dos fabricantes de equipamentos para plantas \\
\hline & & Incentivo fiscal com redução de imposto de importação \\
\hline & \multirow{2}{*}{$\begin{array}{l}\text { Risco de adoção de tecnologias já } \\
\text { superadas ou que não garantam } \\
\text { níveis adequados de emissões }\end{array}$} & $\begin{array}{l}\text { Avaliar tecnicamente as tecnologias disponíveis (Europa, China, EUA) em termos de eficiência e garantia de } \\
\text { controle de emissões }\end{array}$ \\
\hline & & Adotar boas prática/recomendações previstas na BAT (EUROPEAN COMMISSION, 2019) \\
\hline \multirow{4}{*}{$\begin{array}{l}\text { Estudo dos custos } \\
\text { e despesas }\end{array}$} & \multirow{2}{*}{$\begin{array}{l}\text { Falta de experiência e mão de } \\
\text { obra capacitada local }\end{array}$} & Estimular desenvolvimento de tecnologia/engenharia locais \\
\hline & & Contratar especialistas internacionais a longo prazo e lançar programa de capacitação \\
\hline & \multirow{2}{*}{$\begin{array}{l}\text { Variação na heterogeneidade e } \\
\text { sazonalidade nos rsus de entrada }\end{array}$} & Realizar estudo detalhado da características dos resíduos para adoção de tecnologias apropriadas no projeto \\
\hline & & Estabelecer plano de contigência operacional para determinadas faixas de variação \\
\hline \multirow{3}{*}{ Estudo da receita } & $\begin{array}{l}\text { Volatilidade do preço da energia } \\
\text { no mercado }\end{array}$ & $\begin{array}{l}\text { Adotar contratos de fornecimento de energia a longo prazo. Uma demanda constante para a energia gerada } \\
\text { pode consideravelmente compensar os custos da planta }\end{array}$ \\
\hline & \multirow[b]{2}{*}{$\begin{array}{l}\text { Receitas, por si só, podem não } \\
\text { ser suficientes para viabilidade } \\
\text { financeira }\end{array}$} & Avaliar subsídios públicos (concessões financeiras, empréstimos a juros baixos para plantas, redução de impostos) \\
\hline & & $\begin{array}{c}\text { Avaliar a aplicação de: taxas diretas de resíduos a cidadãos; financiamento cruzado de serviços de RSU por meio } \\
\text { de outras taxas ou impostos locais, reembolsos de impostos e aplicação de tarifas especiais para eletricidade } \\
\text { produzida a partir de fontes não convencionais, como RSU }\end{array}$ \\
\hline \multirow[b]{2}{*}{$\begin{array}{l}\text { Avaliação } \\
\text { econômico-social }\end{array}$} & \multirow[b]{2}{*}{ Adequação do arcabouço legal } & $\begin{array}{l}\text { Elaborar/atualizar legislação específica nacional para o licenciamento de instalações de incineração, bem como } \\
\text { os padrões técnicos para orientar os projetos e definir os critérios para a concessão de licenças ambientais. }\end{array}$ \\
\hline & & $\begin{array}{c}\text { Regulamentar legislação nacional que estabeleça as condições para o controle de emissão de poluentes nas } \\
\text { instalações tão restritiva quanto as praticadas pela UE e já previstas na resolução no O79 da Secretaria do Meio } \\
\text { Ambiente do Estado de São Paulo (SMA, 2009) e considerar os investimentos necessários nos projetos de } \\
\text { incineradores para alcance desses níveis limites de emissões }\end{array}$ \\
\hline $\begin{array}{l}\text { Estudo do modelo } \\
\text { de negócio }\end{array}$ & $\begin{array}{l}\text { Seleção de modelo de negócio } \\
\text { adequado }\end{array}$ & $\begin{array}{l}\text { Estimular política de participação pública adequada através das PPPs para atrair investidores privados e } \\
\text { contribui para o sucesso da implementação no país. }\end{array}$ \\
\hline \multirow[b]{2}{*}{ Avaliação financeira } & \multirow{2}{*}{$\begin{array}{l}\text { Lacuna em termos de orientação } \\
\text { para etapa } 8 \text { (avaliação financeira) } \\
\text { da portaria no } 577\end{array}$} & Realizar as etapas complementares da análise financeira apresentadas na Figura 2 \\
\hline & & Avaliar contratação de consultoria especializada para realização da análise financeira \\
\hline
\end{tabular}

PNRS: política nacional de resíduos sólidos; CAPEX: capital expenditure; BAT: best available techniques; RSU: resíduo sólido urbano; UE: união europeia; PPP: parcerias públicoprivadas.

Fonte: Próprio autor

de Gestão Integrada de Resíduos da área atendida e as possíveis variações das características destes resíduos.

Considerando se tratar de um empreendimento de CAPEX muito elevado, aos órgãos de controle cabe assegurar que a tecnologia do projeto seja de última geração, evitando riscos de uma planta ultrapassada que não atenda a padrões rígidos operacionais e de controle de emissões de poluentes, colocando em risco o meio ambiente e a saúde pública. Além disso, com as restrições no investimento público, cada vez mais as PPPs vêm sendo instrumentos de viabilização de projetos de saneamento básico e os EVTEs estruturados são elementos primordiais para a viabilização desses projetos.

Os padrões de referência para a elaboração de EVTEs preconizados na Portaria $\mathrm{n}^{\circ} 557$, juntamente com os itens complementares propostos no artigo para etapa de avaliação financeira, englobam os principais aspectos a serem avaliados para um projeto de implantação de um incinerador. Adicionalmente, para se reduzir os riscos de projetos malsucedidos, é importante avaliar a especificidade da região e realizar uma avaliação cuidadosa dos fatores críticos que dificultam a adoção da tecnologia de incineração, de forma a direcionar os esforços nas ações, conforme sintetizado no Quadro 2, para que se estabeleçam as condições necessárias para uma operação adequada tecnicamente e sustentável financeiramente.

\section{CONTRIBUIÇÃO DOS AUTORES}

Andretti, F. V.: conceituação, investigação, análise formal, escrita - primeira redação. Ferreira, J. A.: conceituação, supervisão, validação, escrita - revisão e edição. Mannarino, C. F.: conceituação, supervisão, validação, escrita — revisão e edição. 


\section{REFERÊNCIAS}

ABRANTES, J. M. C. N. Avaliação técnica e económica da aplicação de sistemas Waste to Energy no tratamento de resíduos urbanos em aglomerados de média e pequena dimensão. Dissertação (Mestrado em Engenharia Civil) - Instituto Superior Técnico de Lisboa, 2016.

AQLAN, F.; ALI, E. M. Integrating lean principles and fuzzy bow-tie analysis for risk assessment in chemical industry. Journal of Loss Prevention in the Process Industries, v. 29, p. 39-48, 2014. https://doi.org/10.1016/j.jp.2014.01.006

BAUMANN, R.; CANUTO, O.; GONÇALVES, R. Economia Internacional. São Paulo: Campus, 2004.

BESCHERER, F. Establisher Life Cycle Concept in the Business Environment, Helsinki University of Technology, 2005

BRASIL. Resolução CONAMA n 316, de 29 de outubro de 2000. Dispõe sobre procedimentos e critérios para o funcionamento de sistemas de tratamento térmico de resíduos. Diário Oficial da União, Brasília, DF, Seção 1. p. 92-95. 20 nov. 2002.

BRASIL. Portaria no 557, de 11 de novembro de 2016. Institui normas de referência para a elaboração de estudos de viabilidade técnica e econômico financeira (EVTE). Diário Oficial da União, Brasília, DF, no 218, Seção 1, p. 129.14 nov. 2016.

BRUNNER, P. H.; RECHBERGER, H. Waste to energy key Element for Sustainable Waste Management. Waste Manage, v. 37, p. 3-12, 2015. https:// doi.org/10.1016/j.wasman.2014.02.003

CARVALHO FILHO, J. S. Manual de Direito Administrativo. 30. ed. São Paulo: Atlas. 2016.

Confederation of European Waste-to-Energy Plants (CEWEP). CEWEP Energy Efficiency Report (Status 2007-2010), 2011. Disponivel em: http:// www.cewep.eu. Acesso em: 12 mar. 2020.

Confederation of European Waste-to-Energy Plants (CEWEP). CEWEP Energy Report III, 2017. Disponível em: https://www.cewep.eu/. Acesso em:12 mar.2O2O.

CHALIKI, P.; PSOMOPOULOS, C. S.; THEMELIS, N. J. WTE plants installed in European cities: a review of success stories. Management of Environmental Quality: An International Journal, v. 27, n. 5, p. 606-620, 2016. http://doi. org/10.1108/MEQ-01-2015-0018

CHIRICO, V. Municipal Waste Treatment Plants. Zurich: Swiss Reinsurance Company. 1996

CHOI, Y:; YE, X:; ZHAO, L.; LUO, A. C. Optimizing enterprise risk management: a literature review and critical analysis of the work of Wu and Olson. Annals of Operations Research, v. 237, n. 1, p. 281-300, 2016. http://doi.org/10.1007/ s10479-015-1789-5

COELHO, S. T. Existing Barriers for WtE in Developing Countries and Policy Recommendations. In: COELHO, S. T.; PEREIRA, A. S.; BOUILLE, D. H.; MANI, S. K.; RECALDE, M. Y.; SAVINO, A. A.; STAFFORD, W. H. L. (Eds.). Municipal Solid Waste Energy Conversion in Developing Countries, 2020. p. 219-234. https://doi.org/10.1016/C2015-0-04596-8

COMMITTEE OF SPONSORING ORGANIZATIONS OF THE TREADWAY COMMISSION (COSO). Gerenciamento de Riscos Corporativos integrado com a Estratégia e o Desempenho. COSO, 2017.
CHINA RESEARCH AND INTELLIGENCE (CRI). Research Report on China's Dairy Industry, 2011-2012, 2014. Disponível em: http://www.cri-report.com/. Acesso em: 13 jan. 2019.

CUCCHIELLA, F.; D'ADAMO, I.; GASTALDI, M. Municipal waste management and energy recovery in an Italian region. Waste Management \& Research, v. 30, p. 1290-1298, 2012. http://doi.org/10.1177/0734242X12462284

DORN, T.; NELLES, M.; FLAMMES, S.; JINMING, C. Waste disposal technology transfer matching requirement clusters for waste disposal facilities in China. Waste Management, v. 32, n. 11, p. 2177-2184, 2012. https://doi.org/10.1016/j. wasman.2012.05.038

ECOPROG. Waste-to-Energy Monitor 16/2020. Ecoprog Krefelder Straße Cologne, Germany, 2020. Disponível em: www.ecoprog.de. Acesso em: 16 ago. 2020

EHRHARDT, M.; BRIGHAM, E. Financial Management, 13. ed. [S.L.] Mc GrawHill. 2010.

ENVIRONMENTAL RESEARCH \& EDUCATION FOUNDATION (EREF) Analysis of MSW Landfill Tipping Fees. Environmental Research \& Education Foundation, 2017. Disponível em: https://erefdn.org/. Acesso em: 09 nov. 2019.

EUROPEAN COMMISSION. Reference Document on the Best Available Techniques (BAT) for Waste Incineration 2019/2010 under Directive 2010/75/ EU of the European Parliament and of the Council, nov 2019. Disponivel em: http://data.europa.eu/eli/dec_impl/2019/2010/oj. Acesso em: 16 ago. 2019.

ENVIRONMENTAL DATA CENTRE ON WASTE (EUROSTAT). Statistics in Focus, 2015. 2016. Disponível em: http://epp.eurostat.ec.europa.eu. Acesso em: 16 ago. 2019

ENVIRONMENTAL DATA CENTRE ON WASTE (EUROSTAT) The EU in the World. Statistical Books. 2020. https://doi.org/10.2785/932944

FINANCIAL ACCOUTING STANDARDS BOARD (FAS). Accounting Standarts: Current Text, Vol. 1. John Wiley: New York, 2002.

FILHO, A.; SOUZA, J. C. F.; GONÇALVES, C. P.; CURY, M. Q. Finanças Corporativa. 12. ed. Rio de Janeiro: FGV, 2016

FIRJAN. Oportunidades de concessões e PPP (parcerias público-privadas) nos estados e municípios do Rio de Janeiro. Nota Técnica, mai. 2019. Disponível em: https://www.firjan.com.br/publicacoes. Acesso em: 02 mai. 2020.

GITMAN, L. J.; ZUTTER, C. J. Princípios de Administração Financeira. 14. ed. São Paulo: Pearson Universidades, 2017. 848 p.

DEUTSCHE GESELLSCHAFT FÜR INTERNATIONALE ZUSAMMENARBEIT (GIZ). Waste-to-energy options in municipal solid waste management: a guide for decision makers in developing and emerging countries. 2017. Disponivel em: https://www.giz.de. Acesso em: 25 abr. 2020.

HITACHI ZOSEN INOVA (HZI). Lucerne (Perlen) / Switzerland Energy-fromWaste plant, Zurich, 2016. Disponível em: https://www.hz-inova.com. Acesso em: 18 nov. $202 \mathrm{O}$.

IEA. Bioenergy: Accomplishments from IEA Bioenergy Task 36: Integrating Energy Recovery into Solid Waste Management Systems (2007-2009). IEA, France, 2013. Disponível em: http://www.ieabioenergytask36.org. Acesso em: 14 jul. 2019. 
LEMES, J. A. B.; CHEROBIM, A. P.; RIGO, C. M. Administração Financeira: princípios, fundamentos e práticas brasileiras. 12. ed. Rio de Janeiro: Elsevier, 2016.

MAISIRI, W.; VAN.DYK, L.; KOCK, J. Financial analysis of waste-to-energy grate incineration power plant for a small city. International Conference on the Industrial and Commercial Use of Energy (ICUE). Cape Town, South Africa: IEEE, 2015

MARTIN, U.; MARTIN, J.; KORALEWSKA, R. WTE, The Martin WTE Technology. In: MEYERS, R. A. (Ed.). Encyclopedia of Sustainability Science and Technology. 1. ed. NY: Springer, 2017. p. 221-283.

MASSARUTTO A. Waste management as a public utility: options for competition in an environmentally-regulated industry. Utilities Policy, v. 15, n. 1, p. 9-19, 2007. http://doi.org/10.1016/j.jup.2006.09.003

MASSARUTTO A. Economic aspects of thermal treatment of solid waste in a sustainable WM system. Waste Management. v. 37, p. 45-57, 2015. https:// doi.org/10.1016/j.wasman.2014.08.024

NZIHOU, A.; THEMELIS, N. J.; KEMIHA, M.; BENHAMOU, Y. Dioxin emissions from municipal solid waste incinerators (MSWIS) in France. Waste Management, v. 32, n. 12, p. 2273-2277, 2012. https://doi.org/10.1016/j.wasman.2012.06.016

O'BRIEN, J. K. Economic benefits of waste-to-energy: jobs creation and community evelopment. MSW Management, v. 23, n. 4, p. 12-19, 2013.

OFFICE FEDERAL DE L'ENVIRONNEMENT (OFEV). Ordonnance sur la protection de lair (OPair) du 16 décembre 1985 (Etat le 11 décembre 2018)

ONU MEDIO AMBIENTE. Perspectiva de la Gestión de Residuos en América Latina y el Caribe. Organização das Nações Unidas, ONU Panamá, 2018.

PASIN, J. A. B.; BORGES, L. F. X. A nova definição de parceria público-privada e sua aplicabilidade na gestão de infraestrutura pública. Banco Nacional de Desenvolvimento Econômico e Social (BNDES.), 2003.

RAGOSSNIG, A. M.; VUJIÉ, G. Challenges in technology transfer from developed to developing countries. Waste Management \& Research. v. 33, p. 93-95, 2015. https://doi.org/10.1177\%2F0734242X15569403

ROSS, S. A.; WESTERFIELD, R. W.; JORDAN, B. D. Fundamentos de Finanças Corporativas. 9. ed. Porto Alegre: McGraw-Hill, 2018.

SCHROEDER, J. T.; SCHROEDER, I.; COSTA, R. P.; SHINODA, C. O custo de capital como taxa mínima de atratividade na avaliação de projetos de investimento. Revista Gestão Industrial, v. 1, n. 2, 2005. http://doi.org/10.3895/ S1808-04482005000200003

SMA. Resolução n 079, de 04/11/2009. Estabelece diretrizes e condições para a operação e o licenciamento da atividade de tratamento térmico de resíduos sólidos em Usinas de Recuperação de Energia (URE). Secretaria de Estado do Meio Ambiente, SP. 2009.

SOÓS, R.; WHITEMAN, A. D.; WILSON, D. C:; BRICIU, C.; NÜRNBERGER, S.; OELZ, B.; GUNSILIUS, E.; SCHWEHN, E. Operator models for delivering municipal solid waste management services in developing countries:Part
B: Decision support. Waste Management \& Research. v. 35, p. 842-862, 2017. https://doi.org/10.1177\%2F0734242X17704717

THEMELIS, N. J.; DIAZ BARRIGA, M. E.; ESTEVEZ, P.; VELASCO, M. G. Guidebook for the application of Waste-To-Energy technologies in Latin America and the Caribbean. Earth Engineering Center: Interamerican Development Bank, New York, NY, 2013.

USINE DE VALORISATION THERMIQUE ET ÉLECTRIQUE DE DECHETS (TRIDEL). (2014) Emissions à la cheminée - tableau comparatif 2017/2018/2019. Disponível em: http://www.tridel.ch/fr/protection-de-lair. Acesso em: 15 ago. 2018

UNIÃO EUROPÉIA (EU). Conselho da União Europeia. Diretiva 2010/75/EC Relativa às emissões industriais. 24 de novembro de 2010.

UNITED NATIONS INDUSTRIAL DEVELOPMENT ORGANIZATION (UNIDO). Industrial Statistics Database, 2019. Industrial Statistics Database. 2019 Disponível em: https://www.unido.org. Acesso em: O4 abr. 2019.

UN ENVIRONMENT. Waste Management: Considerations for informed decision-making. From International Environmental Technology Centre. 2019. Disponível em: https://www.unenvironment.org. Acesso em: 25 dez. 2019

VAN DER HEIJDEN, K. Planejamento por cenários: a arte da conversação estratégica. 2. ed. Porto Alegre: Bookman, 2009.

VERGARA, S. C., TCHOBANOGLOUS, G. Municipal solid waste and the Environment: A global perspective. Annual Review of Environment and Resources. Annual Review of Environment and Resources, v. 37, n. 1 , p. 277-309, 2012. http://doi.org/10.1146/annurev-environ-050511-122532

WALKER, R. Winning with risk management. v. 2. [s.l]: World Scientific, 2013.

WALTERS, D. Supply chain risk management: vulnerability and resilience in logistics. London: Kogan Page, 2007.

WASTE AND RESOURCES ACTION PROGRAMMES (WRAP). Gate Fees 2017/18 Final Report: Comparing the costs of alternative waste treatment options. 2018. Disponível em: http://www.wrap.org.uk. Acesso em: 12 mai. 2020

WORLD ENERGY COUNCIL. World Energy Resources, 2016. Disponivel em https://www.worldenergy.org. Acesso em: 25 abr. 2020.

YAN, M.; WALUYO, J.; AGAMUTHU, P. Challenges for sustainable development of waste to energy in developing countries. Waste Management \& Research, v. 38, p. 229-231, 2020. http://doi. org/10.1177/0734242X20903564

ZENG, M.; LI, C.; ZHOU, L. Progress and prospective on the police system of renewable energy in China. Renewable and Sustainable Energy Reviews, v. 20, p 36-44, 2013. http://doi.org/10.1016/j.rser.2012.11.048

ZHANG, D.; HUANG, G.; XU, Y.; GONG, Q. Waste-to-energy in china: key challenges and opportunities. Energies, v. 8, n. 12, p. 14182-14196, 2015. https://doi.org/10.3390/en81212422 